\title{
El paisaje como recurso didáctico: La Sierra de Andújar como vehículo para conocer la naturaleza de Sierra Morena.
}

(The landscape as an educational resource: The Sierra de Andújar as a way to know the Sierra Morena nature.)

Javier Lara Hinojosa

Universidad de Jaén, España.

Fecha recepción: 22-03-2018

Páginas 76-82

Fecha aceptación: 30-06-2018

\section{Resumen.}

El objetivo de este artículo es poner el valor el concepto del paisaje. Históricamente se ha considerado el paisaje por su aspecto geográfico en sí y se ha olvidado el potencial pedagógico que posé. La observación del entorno que nos rodea nos permite comprender el interés geográfico, ambiental, patrimonial y paisajístico, pero también entender cómo los paisajes han evolucionado según la influencia antrópica o procesos naturales concretos. Para defender la idea de aprender a través de la observación se propone un recorrido por el Parque Natural de la Sierra de Andújar para comprender la naturaleza de toda Sierra Morena, la frontera física y cultural entre la meseta española y el valle del Guadalquivir. Focalizando la atención en enclaves concretos, podremos disfrutar de panorámicas únicas que le otorgan a este reducto montañoso su marcado carácter excepcional.

Palabras clave: Geografía; pinar de repoblación; educación; Sierra de Andújar; geologia; medio ambiente

\section{Abstract.}

The main objective of this article is to ehnance the value of the landscape as a concept. The landscape has been considered historically by his geographic aspect owing to his educational potential has been forgotten. Looking the surroundings we are able to admire their geography, ambient patrimonial and scenic interests, but also to undernstand how the landscapes changes and evolve as a result of the human influence and natural processes. To defend the idea of leargning through observation it is proposed a tour inside the Natural Park of Sierra de Andújar to understand the Sierra Morena nature, which is the physical and cultural boundaries between the Meseta Central and the Guadalquivir valley. Focusing in specific places, we might enjoy looking the unique panoramic views that bring obout this group of mountains uniqueness.

Keywords: Geography; pine forest repopulation; education; Sierra de Andújar; geology; environment 


\section{1.-Introducción.}

La Sierra de Andújar se protegió como Parque Natural el 28 de julio de 1989. Se encuentra en la parte oriental de Sierra Morena y se encuentra íntegramente en el término municipal de Andújar. Aglutina un gran cantidad de unidades paisajísticas distintas y complejas que, si bien es cierto que adquieren ciertos matices locales propios e insustituibles, nos permiten comprender la hidrografía, geología 0 edafología de Sierra Morena. Utilizando los recursos que tenemos disponibles -éstos son, recorriendo las carreteras públicas A-6177 y A-6178 que atraviesan el Parque Natural desde su sector meridional hasta el límite provincial con la provincia de Ciudad Real- podemos disfrutar de un espacio único en la geografía mediterránea cuyo origen se remonta a la Orogenia Hercínica (entre 3,8-2,8 millones de años), cuando se formaron muchos de los principales relieves de la Península Ibérica y de la que aún hoy quedan reductos, como es el caso de Sierra Morena. Es por ello que expondremos tres paradas en un recorrido lineal a través de las dos carreteras para adquirir las nociones básicas con las que comprender la naturaleza de este enclave protegido giennense. Con este estudio se intenta 1) Facilitar el acceso de la población a los recursos y valores paisajísticos existentes en la región. 2) Incentivar el uso del paisaje como recurso didáctico para el aprendizaje de los conceptos básicos de la geografía y así comprender las causas de la configuración actual del territorio.

\section{2.-Geología.}

El punto kilométrico 15 de la carretera A-6177, donde se encuentra el Mirador El Peregrino, es un lugar idóneo por su interés geológico debido a un afloramiento de granitos que se aprecia en el plano medio (orientación este). Se trata de una estribación del batolito de los pedroches por su vertiente oriental. La peculiaridad reside en ser un ejemplo nítido e ilustrativo del modelado típico granítico, que creando una estructura continua de bolos y piedras caballeras, es conocida con el nombre de berrocal. Concretamente encontramos predominio de granitos biotíticos pórfidos, microgranitos biotíticos y leucogranitos. El resultado es una intensa fracturación en un territorio generalmente alomado y poco abrupto debido a la antigüedad de su formación.

Figura 1: Berrocal sobre el Castellón de San Miguel.

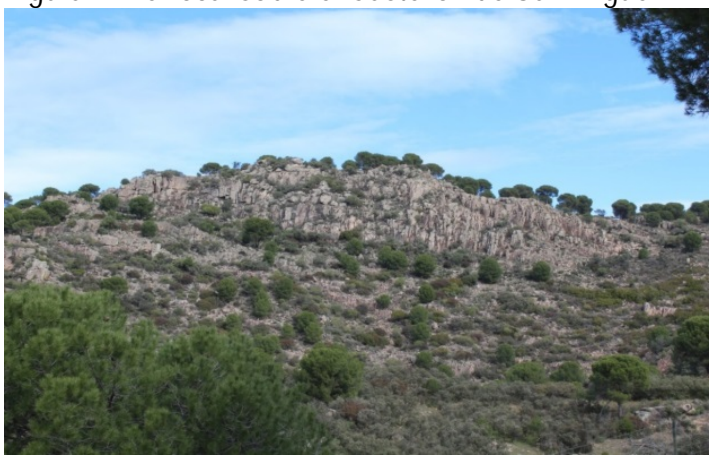


Fuente: Alicia Jiménez González.

Dicho berrocal presenta una altitud superior que la del Mirador, facilitando su visualización. El cerro se conoce como Castellón de San Miguel y se eleva hasta los 608 metros en su parte más alta. La superficie está cubierta con un matorral -figuras 2- poco denso que deja entrever los afloramientos rocosos.

Además, el Mirador constituye el primer lugar que permite contacto visual con El Santuario de la Virgen de la Cabeza situado en el Cerro del cabezo. A pesar de divisarse en la lontananza se puede disfrutar de toda la cuenca visual. La vegetación que coloniza la zona es una vegetación a cabo entre el matorral y elementos aislados de coníferas. En el plano medio se observa la disimetría de coníferas y quercíneas en una proporción similar configurando un bosque mixto. Muchos de los ejemplares de pinos son fruto de las repoblaciones forestales del siglo XX. Posteriormente la naturaleza ha seguido su curso y de ahí que lentamente aflore la vegetación autóctona de la zona: las encinas.

\section{3.-Hidrografía.}

Para comprender cómo la red fluvial vertebra el territorio no hay más que descender hasta el Puente del río Jándula, en el kilómetro 22 de la carretera A-6177. En la intersección de las lomas circundantes discurren arroyos -como el de Valdeinfiernoque viertencsus aguas a la cuenca del río Jándula. Son cursos fluviales estacionales e intermitentes que dependen de la indigencia de precipitaciones (en los meses cálidos y secos la presencia de agua se reduce hasta casi desaparecer por la sequía estival propia de la Sierra Morena oriental). Discurren en perpendicular a las curvas de nivel.

La zona presenta algunas peculiaridades, como el hecho de la alteración de la vegetación de ribera (en pro de la desaparición de la vegetación autóctona) como consecuencia de la acción antrópica, que ha introducido el eucalipto en las lindes del cauce: fueron plantados por su alta capacidad de absorción del agua. Con ello se luchaba contra la amenaza de las ocasionales inundaciones del río Jándula (el principal recurso a la hora de regar la Vega del Guadalquivir) y se evitaba la formación de encharcamientos que propician la propagación del paludismo (Fiebres tifoideas). Aunque el caso del eucalipto no es extensible al resto de Sierra Morena, sí que lo es el hecho de que se sucedan evidencias de acciones antrópicas para ajustar y modificar las vegetaciones de ribera a las necesidades de la población.

La alteración de la cobertura vegetal autóctona también se da lugar con asiduidad en pro de 1) cultivos que demandan grandes cantidades de agua y nutrientes y cuya adaptabilidad es mayor en riberas fluviales, lo que es muy común en los grandes valles peninsulares 0 2) para el mantenimiento de extensiones de pastos en donde las actividades ganaderas y cinegéticas tienen cabida. Ésta última opción es la más desarrollada en los fondos de valle de las cadenas montañosas mediterráneas. "Los pastos pueden tener una larga etapa de aprovechamiento: otoño-invierno primavera, aunque la máxima producción se alcanza en esta última estación Llegado el final de mayo o comienzo de junio, los pastos se agotan hasta después de las primeras lluvias otoñales. La ganadería de la zona está compuesta por vacunos y lanares, que pastan en razón de una cabeza menor o una cabeza de ganado mayor por $506 \mathrm{Ha}$." 
(Evaluación de recursos agrarios. "Mapa de Cultivos y Aprovechamientos", núm. 904, 21).

En esta zona se observan además terrazas fluviales de distinto periodo geológico de formación. La vista se ve favorecida por la construcción de la carretera, que ha provocado el necesario corte transversal del suelo, lo que permite ver su composición litológica. En los fondos de valle encontramos predominio de cantos rodados perfilados por la acción abrasiva del río y depositados en el valle tras ser arrastradas por el agua desde las lomas y cerros circundantes a través de los barrancos y mantos de arroyada a los que anteriormente nos hemos referido.

\section{4.-Biogeografía.}

Continuando por la A-6177 se llega a la A-6178, donde desde el kilómetro 1 y orientando la vista hacia el Sur (S) hacia el Cerro del Cabezo -y gracias a la posición de ventaja que nos otorga estar en un punto más alto- obtenemos una visión magnífica de un claro ejemplo de pinar de repoblación. En toda la Sierra Morena de porte arbóreo serán las quercíneas, encinas (Quercus illex) en su mayoría y alcornoques (Quercus suber) y coníferas adaptadas a la insolación y a la carestía pluviométrica, en especial el pino piñonero (Pinus pinaster) y pino laricio o salgareño (Pinus nigra) en la parte alta de las montañas. En laderas de solana la vegetación arbórea se reduce hasta casi desaparecer; el bosque se degrada y da paso a un matorral dominado por lentisco (Pistacia lentiscus), coscoja (Quercus coccifera) y jara (Cistus ladanifer).

La zona está dominada de forma general por granitos biotíícos pórfidos. El monopolio del granito sólo se ve interrumpido por el afloramiento ocasional de filones de cuarzo, los cuales siempre coinciden con la existencia de fallas.

4.1.- Repoblaciones forestales de Sierra Morena

Repoblar grandes hectáreas de monte fue una política común por el gobierno franquista desde el final de la Guerra Civil en toda Sierra Morena. La repoblación tenía un impacto directo económico al mitigar el desempleo rural de la zona porque suponía la creación de numerosos puestos de trabajo -orientados en la lucha contra incendios, labores de vigilancia y los propios trabajos forestales- $y$ el incremento de aprovechamientos forestales -extracción de madera, obtención de resina, etc.- que servirían para diversificar la economía rural de la zona. Importante resaltar que provocaría indirectamente una restauración ambiental al frenar la erosión en las cuencas de alimentación de los embalses. Los planes de repoblación se llevaron a cabo teniendo en cuenta el potencial económico que presentaba la Sierra de Andújar en el sector cinegético. Fue frecuente ubicar en las laderas de las lomas estas repoblaciones forestales y reservar zonas llanas a la creación de pastizales (para compensar el daño que se le hacía a los ganaderos con la creación de los pinares). Toda política pública de repoblación pilota sobre tres bases, 1) la propiedad de la masa arbórea por parte del Estado 2) la existencia de un marco legislativo que permita la actividad 3) la existencia de un cuerpo de funcionarios públicos y técnicos que lleven a cabo las labores.

El principal inconveniente que se encontró el Gobierno fue que la mayor parte de lo que hoy es el Parque Natural de la Sierra de Andújar era de propiedad privada, pues 
la propiedad pública forestal se había reducido hasta casi desaparecer tras la desamortización de Madoz de 1855. Para poder llevar a cabo sus planes, el Estado creó una serie de mecanismos que le permitieron llevar a cabo sus actuaciones convirtiendo distintas fincas en zonas de urgente y obligatoria repoblación. Se presentaba a los propietarios diferentes fórmulas de obligatoria cooperación, desde las forzadas (consorcio o venta) hasta las forzosas (expropiación). (Guerrero, 2016, pág. 116).

El consorcio, que fue la fórmula más utilizada, consistía en la cesión, por parte del propietario, de la propiedad de la masa arbórea, que pasaba a ser del Estado. El propietario no perdía de ninguna manera la propiedad de la tierra. Para rentabilizar el acuerdo, éste debía de esperar a la explotación de la masa arbórea, cuando recibiría oficialmente el $40 \%$ del valor; a veces esta cantidad era inferior ya que el Estado aplicaba al propietario parte de los gastos derivados del mantenimiento (jornales de desmontes, limpieza del terreno, etc.)

En concreto entre los años 1940-1984 se compraron en Andújar 11.388'82 has - en la Sierra Morena giennense. Esta cantidad sólo es superada por las 16.918'58 has de Montizón-y se adquirieron a través de consorcios 9.246'85 has. Así pues, el avance de la masa arbórea suponía la apuesta decidida por la actividad ganadera y cinegética, al tiempo que se desestimaba la zona para uso agrícola. Esta tendencia lejos de desaparecer, se ha consolidado actualmente. Ya en el año 1970 se contabilizaban según el Servicio Provincial de Ganadería de Jaén un total de 27.145 cabezas de ganado lanar (razas segureña y merina), siendo trashumante y trasterminante; 1.246 cabezas de vacuno de lidia y 236 de vacuno retinta y 1.370 cabezas de ganado caprino. Comparando el sector ganadero con el cinegético, para el mismo año que el caso anterior -temporada 1969/1970- se montearon 17 fincas (29.000 has) que supusieron la venta de 1.165 ciervos (equivale a 4 reses/100 has) en todo el término municipal de Andújar.

Figura 2: Pinar de repoblación con el Santuario de la Virgen de la Cabeza al fondo.

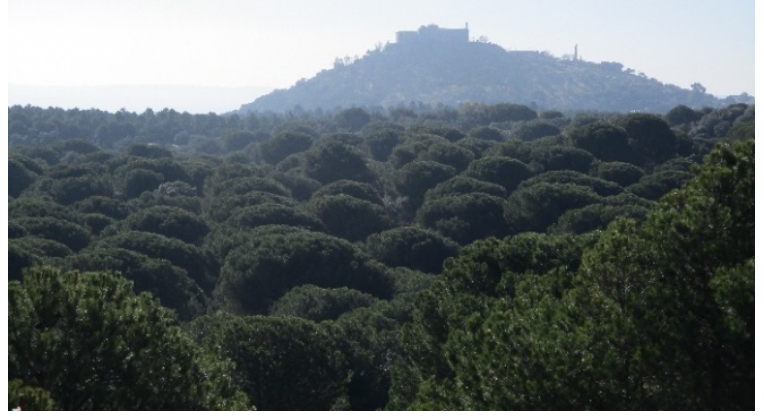

Fuente: Javier Lara Hinojosa

El paisaje actual que se percibe como resultado de la transformación de todos estos grandes latifundios en cotos de caza es el de una extensa propiedad privada homogénea acotada por una alambrada de 1-2 metros para que ganado y animales 
salvajes estén localizados y cercados. Resulta casi imposible recorrer la Sierra de Andújar fuera de las rutas de senderismo catalogadas y del propio trazado de la carretera. Sin embargo no siempre fue así, sino que fue una práctica que se desarrolló paulatinamente -comenzando con el vallado de la finca Los Alarcones (1967)- provocando una distancia insalvable entre la población de Andújar y su propia serranía.

Desde el punto de vista de la fauna, el impulso de la actividad cinegética sirvió para introducir en el territorio especies hasta entonces no presentes en la zona: el gamo 0 el muflón. "El primer coto de caza de la Sierra de Andújar donde se introdujo el muflón fue Los Escoriales" (Guerrero, 2016, pág. 122). Aunque no todo fueron luces, también encontramos grandes sombras de consecuencias irreversibles. La actividad cinegética no sólo se orientaba a venado y jabalí, animales que hoy se encuentran protegidos -el lobo y el lince ibérico- constituían sendos trofeos de caza. Así lo corrobora el trofeo de lince de 22' 70 E (categoría: plata) que consiguió en 1961 el Conde de Caralt en la finca de Lugar Nuevo. De Igual manera, el lobo era codiciado y lo atestiguan los 5 grandes trofeos que se consiguieron en 1971 en Lugar Nuevo: José Sotillo Rubio (42'00 E), Duque de Primo de Rivera (41'00 E), Consuelo Castaño de Matos (40'40 E), Eduardo Barreiros (40'10 E) y Cándido Cortés Durán (38'90 E).

Sin duda las monterías oficiales -y no sólo las batidas de cazadores furtivos- tienen mucho que ver con la actual posición del lince ibérico en peligro de extinción y de la ínfima población del lobo en la Sierra de Andújar.

\section{5.-Metodología.}

La fase teórica se ha completado siempre con una segunda fase práctica. Se han realizado continuas salidas de campo, siempre con material topográfico de apoyo del Instituto Geológico y Minero de España (IGME), Instituto Geográfico Nacional (IGN), y mapas del ejército, con el que identificar las unidades de relieve sobre las que discurre la carretera, así como otros datos a tener en cuenta en cuanto a la red hidrográfica, los cultivos y aprovechamientos del lugar, la composición de los materiales y/o su distribución.

Tras recorrer el itinerario varias veces, buscamos posibles zonas donde poder realizar una parada que nos permita visualizar el paisaje sin comprometer la seguridad tanto del resto de usuarios de la vía como de nosotros mismos. Es en esta fase cuando recurrimos al material fotográfico.

\section{6.-Referencias.}

Busquets, J.; Cortina, A. (2009). Gestión del paisaje. Manual de protección, gestión y ordenación del paisaje. Barcelona: Ariel. ISBN 9788434428904.

Cancer, L. (1999). La degradación y la protección del paisaje. Madrid: Cátedra. ISBN 8437617278. 
Español Echániz, I. (1998). Las obras públicas en el paisaje. Guía para el análisis y evaluación de impacto ambiental en el paisaje. Madrid: Ministerio de Fomento. Centro de Publicaciones.

Español Echániz, I. (2006). Manual de ecología del paisaje. Aplicada la planificación urbana y de infraestructuras. Madrid: Colegio de Ingenieros de Caminos, Canales y Puertos.

Farima, A. (2011). Ecología del Paisaje. Alicante: Universidad de Alicante. ISBN 9788497171670.

Gil Porras, J.A., Sotomayor, F. (1971). Anteproyecto para la ordenación cinegética de la Sierra Morena occidental. Comarca de Andújar. Madrid: Escuela Técnica Superior de Ingenieros de Montes.

Guerrero Crespo, J. M. (2016). Organización administrativa y acción política sobre el recurso caza mayor en España (1939-1975). El caso de la provincia de Jaén. Jaén: Universidad de Jaén.

Ortuño, F., De La Peña, J. (1979). Reservas y cotos nacionales de caza. Madrid: Colección Naturaleza Española. INFOCA. Región Mediterránea. v.4.

Junta de Andalucía (2008). Guía de carreteras de Andalucía. Sevilla: Consejería de Obras públicas y Vivienda. Anexo VIII.

Nogué, J. (2009). Entre paisajes. Barcelona: Àmbit Servicios Editoriales, SA.

Schelle, K., López Silvestre, F., Hernández, I. (2013). El arte de pasear. Madrid: Díaz \& Pons.

Schelle, K., López Silvestre, F. and Hernández, I. (2013). El arte de pasear. Madrid. Díaz \& Pons. 